\title{
Supercapacitor fractional model - DAQ-based measurements of frequency characteristics and error computation
}

\author{
Marcin Sowa ${ }^{1, *}$, Agnieszka Jakubowska-Ciszek ${ }^{1}$ \\ ${ }^{1}$ Silesian University of Technology, Faculty of Electrical Engineering, Akademicka 2A, 44-100 Gliwice, Poland
}

\begin{abstract}
The study concerns the modelling of a supercapacitor. The frequency characteristics are selected as the measurement basis for the study. The characteristics are obtained through a measurement setup featuring a data acquisition (DAQ) device and an arbitrary function generator (AFG). After the characteristics had been obtained - a parameter estimation procedure is executed. The response of the model is then compared with the measurement results. An error definition determining the agreement of the frequency characteristics is introduced. It takes into account both the real and imaginary parts of the obtained impedances.
\end{abstract}

\section{Introduction}

Supercapacitors are devices mainly used in the field of energy storage $[1,2]$. They can provide high power storage in a relatively short amount of time [3]. In recent years a considerable amount of research has been made concerning their potential applications [4]. Therefore, it is valuable to have access to mathematical models, which can accurately reflect their responses in various circuits. The study described in this paper is a part of a research project concerning supercapacitor models applying fractional calculus (where their usefulness has been numerously proven in recent years [5]).

\section{Fractional model of supercapacitor}

The analysis of this paper is a continuation of the study in [6]. Fractional calculus has many other applications also in other fields, like control systems [7]. In circuit theory some studies have shown its usefulness when modelling coils with ferromagnetic cores $[8,9,10]$. As for the elements of fractional calculus, there are many definitions of derivatives and integrals [11]. This study only concerns the application of the Caputo fractional derivative [12]:

$$
{ }_{0} \mathrm{D}_{t}^{\alpha} x(t)=\frac{1}{\Gamma(1-\alpha)} \int_{0}^{t} \frac{x^{(1)}(\tau)}{(t-\tau)^{\alpha}} \mathrm{d} \tau .
$$

where the derivative of order $\alpha \in[0,1]$ is considered; $x^{(1)}(t)$ is the first time derivative of the variable $x(t)$. The object for the study is a BIGCAP@ supercapacitor with the nominal parameters of $5.5 \mathrm{~V}$ and $0.22 \mathrm{~F}$ [13]. The model selected for the study is a generalization of two simple models often appearing in literature [14]. The model consists of a fractional capacitor and three linear resistances (Fig. 1).

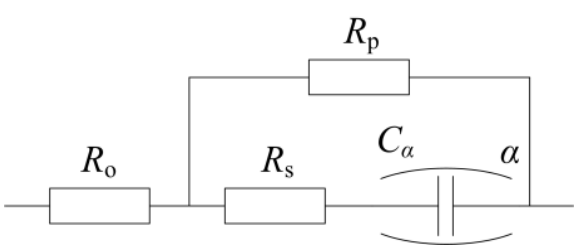

Fig. 1. Applied supercapacitor model including a fractional capacitor element (the symbol has been first introduced in [15]).

\section{The measurement setup}

It has been determined that the frequency characteristics of the supercapacitor will provide the basis for the modelling in order to capture its behaviour for various voltage and current change rates. Such an approach is common and has been applied in studies by other authors [16]. The measurement setup consists of a data acquisition device and an arbitrary function generator (the details on the applied devices is given in [6]). An original algorithm has been applied for the obtainment of the impedances of the supercapacitor for selected frequencies in the range $f \in[0.1,500] \mathrm{Hz}$. it similar to that applied in [9] for coils with ferromagnetic cores (with some exceptions explained with detail in [6]). The setup is depicted in Fig. 2.

\section{Acquired frequency characteristics}

A fragment of the frequency characteristics (up to $f=50$ $\mathrm{Hz}$ where the changes are still significant) is depicted in Fig. 3. An estimation procedure has been completed in order to obtain the fractional capacitor model. The obtained parameters are: 


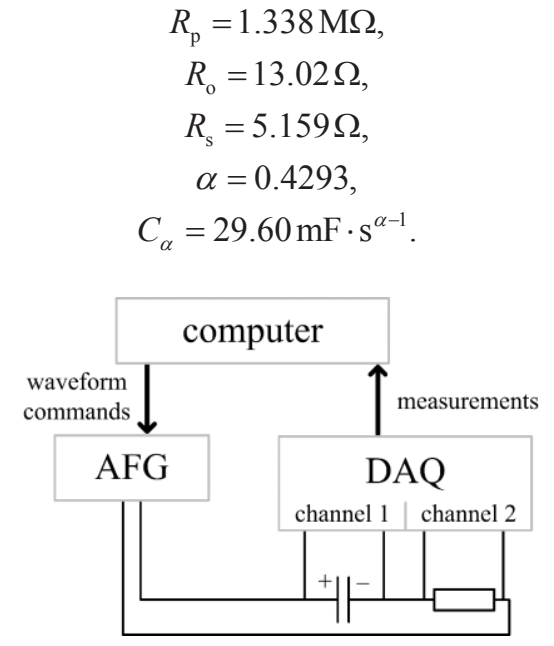

Fig. 2. The applied measurement setup (DAQ - data acquisition device, $\mathrm{AFG}$ - arbitrary function generator).

The frequency response of the model has also been added to Fig. 3.

\section{Model verification - error computation}

In order to verify the correctness of the model an error is defined, which has the following form:

$$
e=\sqrt{\frac{\sum_{j=1}^{N} F_{j}}{N}} .
$$

where $N$ is the number of the points obtained on the frequency characteristics (e.g. the number of actual frequencies for which the impedance has been obtained) and $F_{j}$ is the following value obtained for each selected frequency:

$$
F_{j}=\left(\frac{\operatorname{Re}\left(\underline{Z}_{\mathrm{s} j}\right)-\operatorname{Re}\left(\underline{Z}_{j}\right)}{\left|\underline{Z}_{j}\right|}\right)^{2}+\left(\frac{\operatorname{Im}\left(\underline{Z}_{\mathrm{s} j}\right)-\operatorname{Im}\left(\underline{Z}_{j}\right)}{\left|\underline{Z}_{j}\right|}\right)^{2}, \text { (3) }
$$

with $\underline{Z}_{j}$ being the referential impedance for the selected frequency $f_{j}$, while $\underline{Z}_{\mathrm{s} j}$ is the impedance for that frequency obtained through simulations (i.e. the model response). The value of $e$ obtained for this study is equal to $1.358 \cdot 10^{-2} \%$.

\section{Conclusions}

A modelling study has been performed for a selected supercapacitor. The frequency characteristics have been obtained through a specially prepared setup (Fig. 2). The model selected for the study is one featuring a fractional capacitor (Fig. 1). After an estimation procedure had been completed (in order to obtain the parameters of the model) it has been determined that the model can accurately resemble the frequency characteristics, which was both visible when observing the characteristics (Fig. 3 ) and through error computations (Section 5).

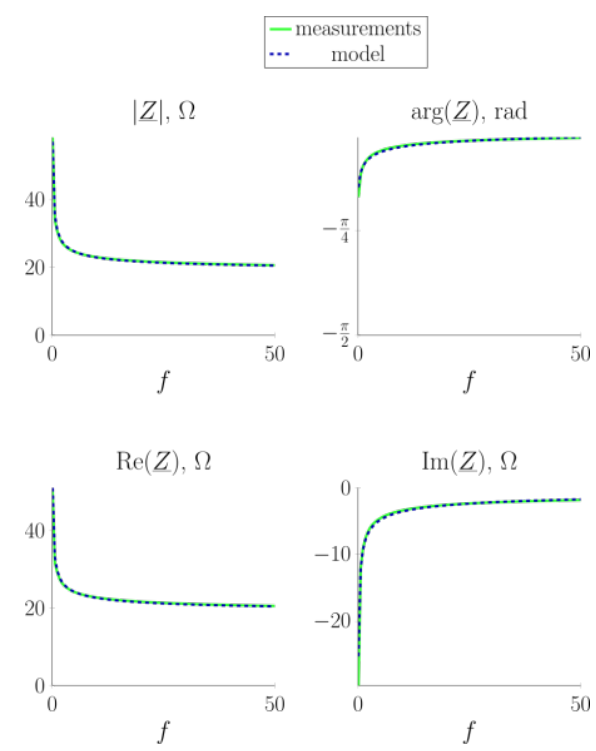

Fig. 3. Comparison of the frequency response of the supercapacitor with that of the applied model.

\section{References}

1. L. Kasprzyk, Przegląd Elektrotechniczny 95, 3 (2019).

2. A. Tomczewski, L. Kasprzyk, Applied SciencesBasel 8, 9 (2018).

3. K. Sahay, B. Dwivedi, Journal of Electrical Systems 5, 4 (2009).

4. A. González, E. Goikolea, J.A. Barrena, R. Mysyk, Renewable and Sustainable Energy Reviews 58 (2016).

5. A. Allagui, T.J. Freeborn, A.S. Elwakil, M.E. Fouda, B.J. Maundy, A.G. Radwan, Z. Said, M.A. Abdelkareem, Journal of Power Sources 400 (2018).

6. M. Sowa, A. Jakubowska-Ciszek, Pozn. Univ. Technol. Acad. J., Electr. Eng. 97 (2019).

7. A. Jajarmi, D. Baleanu, Journal of Vibration and Control 24, 12 (2018).

8. Ł. Majka, Bull. Pol. Acad. Sci., Tech. Sci. 66, 4 (2018).

9. M. Sowa, 2018 International Interdisciplinary PhD Workshop (IIPhDW), IEEE (2018).

10. Ł. Majka, M. Klimas, Electr. Eng. (2019).

11. E. Capelas De Oliveira, J.T. Machado, Mathematical Problems in Engineering (2014).

12. M. Caputo, Geophys. J. Int. 13, 5 (1967).

13. http://www.bigcap.net/en/article/389.html

14. W. Mitkowski, P. Skruch, Bull. Pol. Ac.: Tech. Sci. 61, 3 (2013).

15. M. Sowa, Circuits, Systems and Signal Processing 37, 11 (2018).

16. T.J. Freeborn, B. Maundy, A.S. Elwakil, Fractionalorder models of supercapacitors, batteries and fuel cells: a survey, Mater Renew Sustain Energy, 4:9 (2015) 